\section{Fibroblast growth factor 19, a double-edged sword}

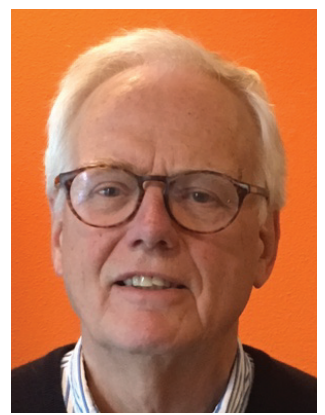

Peter LM Jansen*

\author{
"FXR and FGF19 play a critical role in \\ protecting the liver against bile salt \\ toxicity."
}

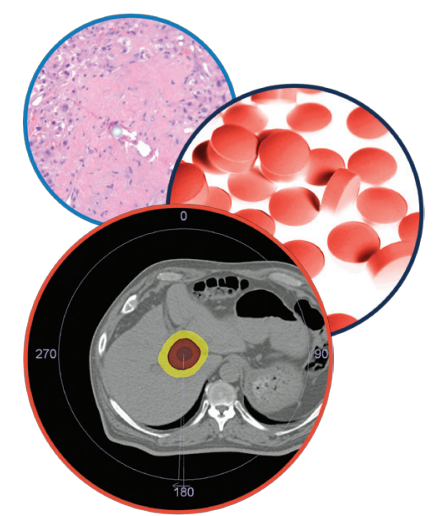

Hepatic Oncology

First draft submitted: 6 April 2017; Accepted for publication: 12 May 2017; Published online: 6 July 2017

The liver is specialized in handling bile salts. Bile salts are needed for bile formation and the digestion of fats in the intestine. It is this digestive function that makes bile salts cytotoxic. At concentrations in the millimolar range bile salts act as detergents and at lower concentrations they are proapoptotic, proinflammatory and cause necrosis [1]. Therefore, when during evolution changes in nutritional habits demanded the digestion of fats as a source of calories, bile salts with detergent properties were needed and mechanisms evolved to limit the toxicity of bile salts in the liver. The farnesoid X-receptor (FXR) and FGF19 play a critical role as protectors of the liver.

FXR is expressed in many organs and cell types, including hepatocytes and ileum [2]. FGF19 is expressed in the ileum, gallbladder epithelium and, under cholestatic conditions, also in human liver [3,4]. FGF19 expression is controlled by FXR. The FGF19 gene on chromosome locus $11 \mathrm{q} 13$ encodes a $22 \mathrm{kDa}$ protein and member of a family of FGFs that are important regulators of organogenesis during fetal development. FGF19, FGF21 and FGF23 are different from the other family members as they lack the heparin-binding domain that tethers FGFs to their cells of origin. In contrast to the other FGFs, FGF19, FGF21 and FGF23 are produced life-long. FGF19 is produced in the ileum and acts in the liver through its receptor FGFR4 and coreceptor $\beta$-klotho. Binding of FGF19 to FGFR 4 activates the phosphorylation of ERK1, ERK2 and Stat-3, and this affects downstream target genes with regulatory roles in gluconeogenesis, lipogenesis, bile salt synthesis and proliferation. Protection from bile salt-mediated cytotoxicity largely depends on the FXR- and FGF19mediated downregulation of CYP7A1, the rate-limiting enzyme in de novo bile salt synthesis. For instance, liver regeneration in $\mathrm{FXR}^{-/}$mice is hampered by bile salt toxicity resulting from nonrepressed bile salt synthesis [5]. Additional cytoprotection during regeneration and cholestasis is provided by downregulation of NTCP (the basolateral sodium-dependent

*Department of Gastroenterology \& Hepatology, Academic Medical Center, Amsterdam, The Netherlands; 


\section{"Treatment with FXR-agonists leads to prolonged elevations of serum FGF19 levels. Whether or not this causes a cancer risk, needs to be studied."}

taurocholate cotransporting protein for hepatic uptake of bile salts) and upregulation of BSEP (the ATP-dependent canalicular bile salt export pump). A third line of defense is established by FXR- and FGF19-mediated upregulation of the basolateral export pumps MRP3, MRP4 and OST $\alpha \beta$, mediating the efflux of bile salts from hepatocytes when canalicular secretion is impaired or halted.

We have reported increased FGF19 serum levels in patients with extrahepatic obstructive cholestasis [4]. FGF19 mRNA in the liver of these patients is increased indicating that FGF19 in serum in part may be produced in the liver, and this suggests that cholestasis in the liver may stimulate FGF19 production by cholestatic hepatocytes.

The beneficial effects of FXR- and FGF19signaling are supported by studies in FXR1- knock out mice. These mice spontaneously develop liver tumors. This can be prevented by the expression of an FXR-transgene in the intestine [6-8]. This suggests a protective role of Fgf15 (the mouse homolog of human FGF19) toward liver cancer. The mechanism behind tumor development in $\mathrm{FXR}^{-/}$mice most likely results in part from an unrestricted, unrepressed bile salt synthesis by upregulated Cyp7A1. Increased intracellular bile salts have proinflammatory and direct or indirect tumor-promoting actions.

However, the story becomes more complicated by considering the following. Transgenic ectopically FGF19 expressing mice have lasting high serum levels of FGF19. At an age of 10-12 months, glutamine-synthase, $\beta$-catenin positive hepatocellular carcinomas (HCCs) developed in $80 \%$ of female and $22 \%$ of male mice [9]. In line with this, Uriarte et al. showed that CCl4-diethylnitrosamine-treated $\mathrm{Fgf} 15^{-/}$mice develop fewer tumors than identically treated Fgf15 $5^{+/+}$mice [10]. This suggests that Fgf15 in mice may be involved in HCC development, either directly or indirectly as a co-factor. Thus, while Fgf15 prevents spontaneous tumor growth, Fgf15 stimulates chemically induced tumor development, seemingly contrasting observations. These models obviously represent different oncogenic pathways. From these studies it is difficult to predict if in humans longterm elevation of FGF19 will be protective or represents a cancer risk.

More direct evidence for human FGF19 as a carcinogenic factor comes from studies in which the metabolic and procarcinogenic actions of
FGF19 have been dissected on a molecular level. A bioengineered FGF19 variant, in which a set of amino acids has been replaced, has retained its ERK1,2-dependent metabolic activity but has lost its STAT3-dependent procarcinogenic action [11]. Thus while the transgenic expression of FGF19 ameliorated inflammation and attenuated fibrosis, FGF19 expression was also associated with development of liver tumors in various mouse models ( $\mathrm{mdr} 2^{-/-}, \mathrm{db} / \mathrm{db}$, rasH2 mice and mice on a high fat diet). In contrast, when the engineered FGF19 variant was expressed in these mouse models, the same metabolic effects were seen but no cancer formation was observed [12].

For more direct evidence that FGF19 may be carcinogenic, one has to consider the molecular signature of human HCC. In about $14 \%$ of HCCs, the Wnt/ $\beta$-catenin/FGF19 pathway is amplified $[13,14]$. In these tumors FGF19 may stimulate tumor growth via autocrine or paracrine mechanisms. Interestingly the Wnt/ $\beta$ catenin pathway is linked to cholestasis. This is in line with observations that $\beta$-catenin $\mathrm{KO}$ mice are cholestatic [15]. Thus, one may speculate that $\beta$-catenin is needed for BSEP expression. Together these observations indicate that FGF19 in HCC may be increased directly by overexpression or gene amplification as well as indirectly by local cholestasis. Figure 1 shows how FGF19 could induce tumor growth.

The pharmaceutical industry has turned the FGF19 story into a druggable option. Novel drugs to interfere with this pathway include FGF-ligand traps, FGFR antagonists like Brivanib, TSU-68, BIBF 1120 and E7080, the FGFR4 antagonist BLU 9931, FGFR mAbs and the tyrosine kinase inhibitors sorafenib and sunitinib. Most of these agents (except sorafenib that is registered for clinical use) are still in clinical development. Given the physiological role of FGF19 signaling it is clear that these drugs may have side effects (liver toxicity and diarrhea) [16].

Additional evidence for the role of FGF19 signaling in HCC tumor development comes from the tumor-preventing action of the selective FGFR4 antagonist BLU9931. This agent reduced tumor development in a mouse HCC model with implanted FGF19-producing, FGFR4-expressing liver cells [17].

These findings indicate that FGF19 may be involved in tumor development, at least in a subset of HCCs, and reveal the dark side of FGF19. However, this does not necessarily mean that the natural production of FGF19, or drug-induced 


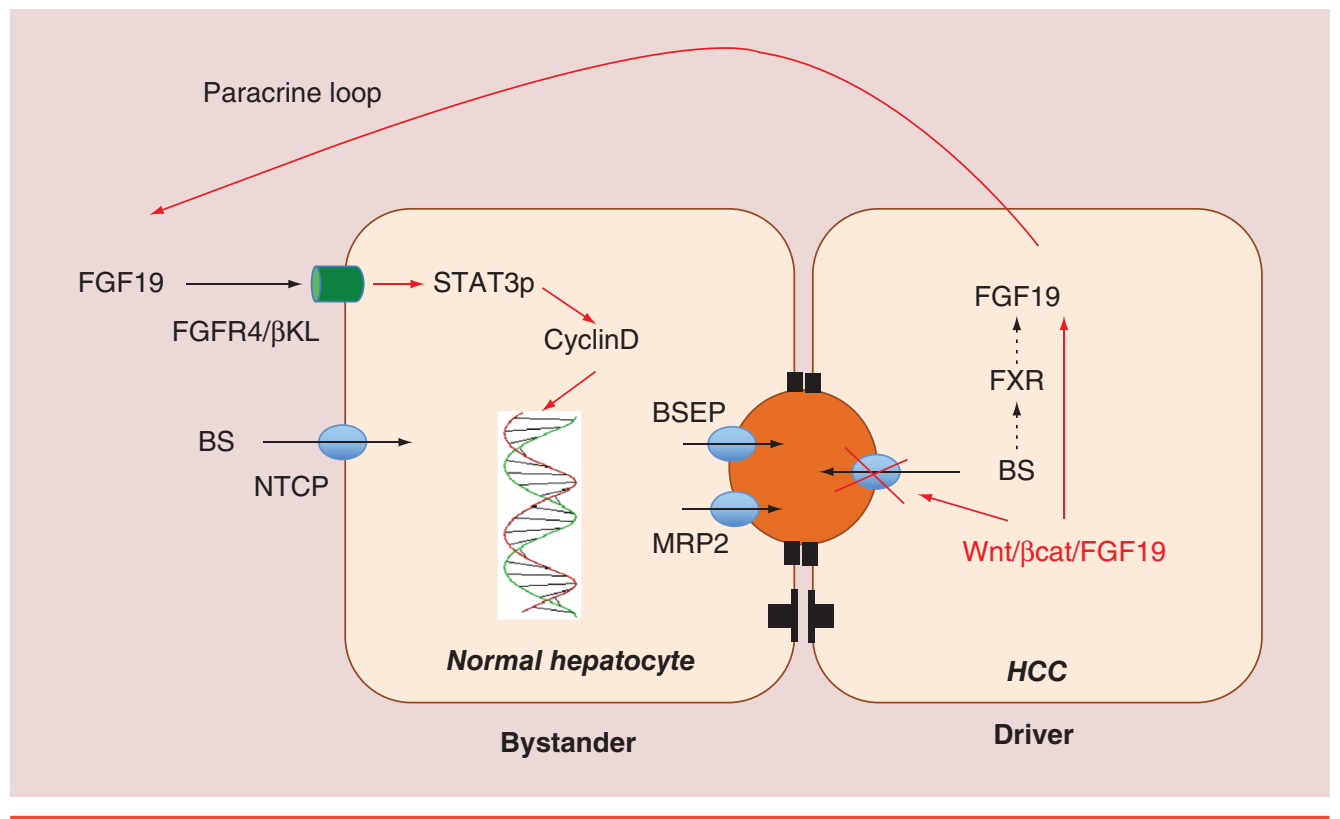

Figure 1. Wnt/ $\beta$-catenin HCCs directly express FGF19 by gene amplification and indirectly as a result of a cholestatic phenotype. One may speculate that the cholestasis results from repression or inhibition of the canalicular bile salt pump BSEP. FGF19 produced by these HCC cells may have a proproliferative effect on neighboring hepatocytes creating 'drivers' and 'bystanders'.

BSEP: Bile salt export pump; HCC: Hepatocellular carcinoma.

elevations of serum FGF19, presents a cancer risk but they show that caution is warranted. There might be carcinogenic effects of FGF19 that are concentration-dependent and occur after prolonged exposure.

Liver cirrhosis is considered as a premalignant condition. FGF19 needs to be studied in cirrhotic patients to find out if elevated FGF19 may be an HCC risk factor in cirrhotic patients. This is particularly relevant for patients with primary sclerosing cholangitis (PSC). This is a chronic cholestatic liver disease with a $20 \%$ life-time risk for developing bile duct cancer. Chronically increased bile salts and FGF19 levels may constitute risk factors that may be present years before the development of cancer.

In conclusion, these studies indicate that FGF19 may play a role in human FGF19/FGFR4 expressing HCCs. They do not provide the unequivocal proof that FGF19 is indeed involved in carcinogenesis in patients with liver cirrhosis or PSC, nor do they provide evidence that FXRagonists, while causing chronically elevated FGF19 levels, pose a cancer risk. However, caution is needed when FXR-agonists are prescribed for durations longer than a few weeks, in particular in patients in whom the cancer risk is already elevated such as in patients with liver cirrhosis or PSC.

\section{Financial \& competing interests disclosure}

The author is on the advisory board of Intercept and on a data management board of a RCT by NGM Pharmaceuticals. The author has no other relevant affiliations or financial involvement with any organization or entity with a financial interest in or financial conflict with the subject matter or materials discussed in the manuscript apart from those disclosed.

No writing assistance was utilized in the production of this manuscript.

\section{References}

1 Jansen PL, Ghallab A, Vartak N et al. The ascending pathophysiology of cholestatic liver disease. Hepatology 65, 722-738 (2017).

2 Inagaki T, Choi M, Moschetta A et al. Fibroblast growth factor 15 functions as an enterohepatic signal to regulate bile acid homeostasis. Cell Metab. 2, 217-225 (2005).

Zweers SJ, Booij KA, Komuta M et al. The human gallbladder secretes fibroblast growth factor 19 into bile: towards defining the role of fibroblast growth factor 19 in the enterobiliary tract. Hepatology 55, 575-583 (2012).

4 Schaap FG, van der Gaag NA, Gouma DJ, Jansen PL. High expression of the bile salt-homeostatic hormone fibroblast growth 
factor 19 in the liver of patients with extrahepatic cholestasis. Hepatology 49, 1228-1235 (2009).

5 Huang W, Ma K, Zhang J et al. Nuclear receptor-dependent bile acid signaling is required for normal liver regeneration. Science 312, 233-236 (2006).

6 Yang F, Huang X, Yi T, Yen Y, Moore DD, Huang W. Spontaneous development of liver tumors in the absence of the bile acid receptor farnesoid X receptor. Cancer Res. 67, 863-867 (2007).

7 Kim I, Morimura K, Shah Y, Yang Q, Ward JM, Gonzalez FJ. Spontaneous hepatocarcinogenesis in farnesoid $\mathrm{X}$ receptor-null mice. Carcinogenesis 28, 940-946 (2007).

8 Degirolamo C, Modica S, Vacca M et al. Prevention of spontaneous hepatocarcinogenesis in farnesoid $\mathrm{X}$ receptor-null mice by intestinal-specific farnesoid X receptor reactivation. Hepatology 61, 161-170 (2015)
9 Nicholes K, Guillet S, Tomlinson E et al. A mouse model of hepatocellular carcinoma: ectopic expression of fibroblast growth factor 19 in skeletal muscle of transgenic mice. Am. J. Pathol. 160, 2295-2307 (2002).

10 Uriarte I, Latasa MU, Carotti S et al. Ileal FGF15 contributes to fibrosis-associated hepatocellular carcinoma development. Int. J. Cancer 136, 2469-2475 (2015).

11 Zhou M, Wang X, Phung V et al. Separating tumorigenicity from bile acid regulatory activity for endocrine hormone FGF19. Cancer Res. 74, 3306-3316 (2014).

12 Zhou M, Learned RM, Rossi SJ, DePaoli AM, Tian H, Ling L. Engineered fibroblast growth factor 19 reduces liver injury and resolves sclerosing cholangitis in Mdr2deficient mice. Hepatology 63, 914-929 (2016).

13 Zucman-Rossi J, Villanueva A, Nault JC, Llovet JM. Genetic landscape and biomarkers of hepatocellular carcinoma. Gastroenterology 149, 1226-1239 e1224 (2015).
14 Ahn SM, Jang SJ, Shim JH et al. Genomic portrait of resectable hepatocellular carcinomas: implications of RB1 and FGF19 aberrations for patient stratification. Hepatology 60, 1972-1982 (2014).

15 Behari J, Yeh TH, Krauland L et al. Liver-specific beta-catenin knockout mice exhibit defective bile acid and cholesterol homeostasis and increased susceptibility to diet-induced steatohepatitis. Am. J. Pathol. 176, 744-753 (2010).

16 Pai R, French D, Ma N et al. Antibodymediated inhibition of fibroblast growth factor 19 results in increased bile acids synthesis and ileal malabsorption of bile acids in cynomolgus monkeys. Toxicol. Sci. 126, 446-456 (2012).

17 Hagel M, Miduturu C, Sheets M et al. First selective small molecule inhibitor of fgfr 4 for the treatment of hepatocellular carcinomas with an activated FGFR4 signaling pathway. Cancer Discov. 5, 424-437 (2015). 